\title{
Synthesis and Bioevaluation of 3-(substitutedphenyl)-1- (4-hydroxyphenyl)-2-propen-1-ones and their Carbamate Derivatives against Root - Knot Nematode (Meloidogyne javanica)
}

\author{
SUMONA KUMARI*, RAJVIR SINGH and R.K. WALIA \\ Department of Chemistry \& Physics, CCS Haryana Agricultural University, Hisar - 125004, India. \\ ${ }^{\star}$ Corresponding author E-mail: sumonasghanghas@gmail.com \\ http://dx.doi.org/10.13005/ojc/300346
}

(Received: May 30, 2014; Accepted: July 08, 2014)

\begin{abstract}
Synthesis of 3-(4-chloro/methyl/nitro/methoxy/3,4,5-trimethoxy/2,4-dichloro/ 4-bromo/ 3, 4-dimethoxy/2, 4-dimethoxy/3-bromo/ 2-chloro/ 2-methoxy phenyl)-1-(4-hydroxyphenyl)-2-propen-1one (14-25) has been carried out from substituted benzaldehyde (1-12) and 4-hydroxy acetophenone (13). The condensation of synthesized 2-propen-1-ones (14-25) with phenyl isocyanate (26) gave 4-[3-(4-chloro/ methyl/nitro/methoxy/3, 4, 5-trimethoxy/2, 4-dichloro/4-bromo/3, 4-dimethoxy/2, 4-dimethoxy/3-bromo phenyl) prop-2-enoyl] phenyl phenylcarbamate (27-36). The synthesized compounds were characterized on the basis of analytical and spectral data. All the compounds were evaluated for their nematicidal activity in- vitro against second stage juveniles $\left(\mathrm{J}_{2}\right)$ of root knot nematode (Meloidogyne javanica). Incorporation of carbamoyloxy moiety in 2-propen-1-ones enhanced the activity. Irrespective of the concentration, compounds 3-(4-methoxyphenyl)-1-(4hydroxyphenyl)-2-propen-1-one (17) and 4-[3-(4-methoxyphenyl) prop-2-enoyl] phenyl phenyl carbamate (30) have shown maximum nematode mortality i.e. $30 \%$ and $51.8 \%$.
\end{abstract}

Key words: Substituted-2-propen-1-one, Carbamate, Nematicidal activity,

Root-knot nematode, Meloidogyne javanica.

\section{INTRODUCTION}

Plants stunted or diseased by nematode related activity are likely to produce reduced yields and loss in quality and quantity of the produce $^{1}$. Inspite of considerable damage caused by nematodes to various agricultural crops, till today very few nematicides are available for use. Chalcones are natural biocides and are well known intermediates for the synthesis of various organic heterocycles. The presence of a reactive, \pm 2 unsaturated keto function in chalcones is found to be responsible for their antimicrobial activity ${ }^{2}$. In present study, we have utilized 3-(substituted phenyl)-1-(4hydroxyphenyl)-2-propen-1-ones as starting material for the synthesis of carbamates. The carbamates are derivatives of carbamic acid, $\mathrm{HOC}(\mathrm{O}) \mathrm{NH}_{2}$ and are well known class of insecticides. Carbamates were originally extracted from the calabar bean, which grows in west Africa. 
Carbamates as a class are not generally persistent in the environment ${ }^{3}$. Carbamates are effective nematicides by virtue of their ability to inhibit acetyl cholinesterase (AchE) in the nervous system and thereby, disrupt nervous transmission at that location ${ }^{4}$. In view of the diverse type of biological activities shown by carbamates, their importance as agrochemicals and as a part of our ongoing new nematicide development programme ${ }^{5-7}$, it was thought of interest to synthesize and evaluate nematicidal activity of substituted 4-[3-(substitutedphenyl) prop2-enoyl]phenyl phenyl carbamates.

\section{EXPERIMENTAL}

\section{Materials \& Methods}

The melting points were determined in open capillaries on a Ganson electrical melting point apparatus and are uncorrected. Homogeneity of the compounds was routinely checked on silica gel-G TLC plates using ethyl acetate: hexane (3:7) as irrigant. IR spectra were recorded on "Perkin Elmer FTIR" spectrophotometer in $\mathrm{KBr}$ and frequencies are expressed in $\mathrm{cm}^{-1}$. The NMR spectra were recorded on "Bruker AC-400-F" (400MHz) NMR spectrophotometer in $\mathrm{CDCl}_{3}$ or $\mathrm{DMSO}-\mathrm{d}_{6}$ using tetramethylsilane (TMS) as internal reference. The chemical shift values are expressed in ' (ppm) units while $\mathrm{J}$ values in $\mathrm{Hz}$ and are compatible with the assigned structures. The elemental analyses were within $\pm 0.4 \%$ of that of evaluated values. Only those spectral data have been mentioned which have a direct bearing on the assignment of the structures and are discussed here.

\section{Chemistry}

3-(4-chlorophenyl)-1-(4-hydroxyphenyl)-2propen-1-one (14)

A solution of 4-chlorobenzaldehyde $(2.8 \mathrm{gm}$, $20 \mathrm{mmol}$ ) and 4-hydroxyacetophenone (2.72gm, $20 \mathrm{mmol})$ in ethanol $(125 \mathrm{ml})$ was taken in round bottom flask placed in ice bath and to it added an aqueous sodium hydroxide solution $(20 \mathrm{ml}, 20 \%)$ slowly with stirring. It was further stirred for $3 \mathrm{~h}$ in ice bath. The mixture was then diluted with cold distilled water and neutralized with $2 \mathrm{~N} \mathrm{HCl}$. The solid that separated out was filtered, washed with cold water and crystallized from ethanol to afford 14. Yield 4.10g (79.3\%), m.p. $213-214^{\circ} \mathrm{C}$ (Lit. $\left.216^{\circ} \mathrm{C}\right)^{8}$.

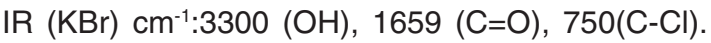

${ }^{1} \mathrm{HNMR}\left(\mathrm{DMSO}-\mathrm{d}_{6}\right): 7.19\left(\mathrm{~d}, \mathrm{~J}=8.0 \mathrm{~Hz}, 2 \mathrm{H}, \mathrm{C}_{3}-\mathrm{H} \&\right.$ $\left.\mathrm{C}_{5}-\mathrm{H}\right), 7.42(\mathrm{~d}, \mathrm{~J}=16.0 \mathrm{~Hz}, 1 \mathrm{H}, \mathrm{COCH}=\mathrm{CH}), 7.67(\mathrm{~d}$, $\left.\mathrm{J}=8.0 \mathrm{~Hz}, \mathrm{C}_{3}-\mathrm{H} \& \mathrm{C}_{5}-\mathrm{H}\right), 7.69\left(\mathrm{~d}, \mathrm{~J}=8.0 \mathrm{~Hz}, 2 \mathrm{H}, \mathrm{C}_{2}-\mathrm{H}\right.$ \& $\left.\mathrm{C}_{6}-\mathrm{H}\right), 7.74(\mathrm{~d}, \mathrm{~J}=16.0 \mathrm{~Hz}, 1 \mathrm{H}, \mathrm{COCH}=\mathrm{CH}), 7.95(\mathrm{~d}$, $\left.\mathrm{J}=8.0 \mathrm{~Hz}, 2 \mathrm{H},{ }^{\prime} \mathrm{C}_{2}-\mathrm{H} \& \mathrm{C}_{6}-\mathrm{H}\right)$. Analysis found: $\mathrm{C}, 69.24$; $\mathrm{H}, 4.60 ; \mathrm{Cl}, 13.38 \% ; \mathrm{C}_{15} \mathrm{H}_{11} \mathrm{Cl} \mathrm{O}_{2}$ Required: C, 69.64; $\mathrm{H}, 4.29 ; \mathrm{Cl}, 13.70 \%$.

Other compounds 15-25 were prepared similarly from 2-12 and 13.

\section{3-(4-methylphenyl)-1-(4-hydroxyphenyl)-2- propen-1-one (15)}

Yield $80 \%$, m.p. $157-158^{\circ} \mathrm{C}$ (Lit. $\left.156^{\circ} \mathrm{C}\right)^{8}$. IR $(\mathrm{KBr}) \mathrm{cm}^{-1}:$ 3128(OH), $1671(\mathrm{C}=\mathrm{O}) .{ }^{1} \mathrm{HNMR}$ $\left(\mathrm{CDCl}_{3}\right): 2.38\left(\mathrm{~s}, 3 \mathrm{H}, \mathrm{C}_{4}-\mathrm{CH}_{3}\right) ; 7.01(\mathrm{~d}, \mathrm{~J}=8.0 \mathrm{~Hz}, 2 \mathrm{H}$, $\left.\mathrm{C}_{3}-\mathrm{H} \& \mathrm{C}_{5}-\mathrm{H}\right) ; 7.21(\mathrm{~d}, \mathrm{~J}=16.0 \mathrm{~Hz}, 1 \mathrm{H}, \mathrm{CO}-\mathrm{CH}=\mathrm{CH})$; 7.25(d, J=8.0Hz, 2H, $\left.\mathrm{C}_{3}{ }^{6}-\mathrm{H} \& \mathrm{C}_{5}{ }^{6}-\mathrm{H}\right) ; 7.50\left(\mathrm{~d}, 2 \mathrm{H}, \mathrm{C}_{2}-\mathrm{H}\right.$ \& $\left.\mathrm{C}_{6}-\mathrm{H}\right) ; 7.52(\mathrm{~d}, \mathrm{~J}=16.0 \mathrm{~Hz}, 1 \mathrm{H}, \mathrm{CO}-\mathrm{CH}=\mathrm{CH}) ; 7.69$ (d, J=8.0Hz, $2 \mathrm{H}, \mathrm{C}_{2}{ }^{\prime}-\mathrm{H} \& \mathrm{C}_{6}{ }^{\prime}-\mathrm{H}$ ). Analysis found : $\mathrm{C}$, 80.35; $\mathrm{H}, 5.80 \% . ; \mathrm{C}_{16} \mathrm{H}_{14} \mathrm{O}_{2}$ Required : C, 80.65; $\mathrm{H}$, $5.92 \%$.

\section{3-(4-nitrophenyl)-1-(4-hydroxyphenyl)-2-propen- 1-one (16)}

Yield $71 \%$, m.p. $204-206^{\circ} \mathrm{C}$ (Lit. $\left.210^{\circ} \mathrm{C}\right)^{8}$. IR $(\mathrm{KBr}) \mathrm{cm}^{-1}: 3162(\mathrm{OH}), 1660(\mathrm{C}=\mathrm{O}), 1572,1344(\mathrm{C}$ $\mathrm{NO}_{2}$ ). Analysis found: $\mathrm{C}, 66.92 ; \mathrm{H}, 3.94 ; \mathrm{N}$, $5.08 \%$. $\mathrm{C}_{15} \mathrm{H}_{11} \mathrm{NO}_{4}$ Required:C, 66.91; $\mathrm{H}, 4.12 ; \mathrm{N}$, $5.20 \%$.

\section{3-(4-methoxyphenyl)-1-(4-hydroxyphenyl)-2-} propen-1-one (17)

Yield $79 \%$, m.p. $177-179^{\circ} \mathrm{C}$ (Lit. 179$\left.181^{\circ} \mathrm{C}\right)^{9}$. IR $(\mathrm{KBr}) \mathrm{cm}^{-1}: 3126(\mathrm{OH}), 1676(\mathrm{C}=\mathrm{O}), 1250$ $\left(\mathrm{C}-\mathrm{OCH}_{3}\right) .{ }^{1} \mathrm{HNMR}\left(\mathrm{CDCl}_{3}\right): 3.82\left(\mathrm{~s}, 3 \mathrm{H}, \mathrm{C}-\mathrm{OCH}_{3}\right)$; $6.93(\mathrm{~d}, \mathrm{~J}=16.0 \mathrm{~Hz}, 1 \mathrm{H}, \mathrm{CO}-\mathrm{CH}=\mathrm{CH}) ; 7.67(\mathrm{~d}$, $\mathrm{J}=16.0 \mathrm{~Hz}, 1 \mathrm{H}, \mathrm{CO}-\mathrm{CH}=\mathrm{CH}) ; 7.54-7.59(\mathrm{~m}, 6 \mathrm{H}$, Ar-H). Analysis found : $\mathrm{C}, 75.38 ; \mathrm{H}, 5.32 \%$. $\mathrm{C}_{16} \mathrm{H}_{14} \mathrm{O}_{3}$ Required:C, 75.57; H, 5.55\%.

\section{3-(3,4,5-trimethoxyphenyl)-1-(4-hydroxyphenyl)-} 2-propen-1-one (18)

Yield $60 \%$, m.p. $221-223^{\circ} \mathrm{C}$ (Lit. $\left.222^{\circ} \mathrm{C}\right)^{8}$.

IR $(\mathrm{KBr}) \mathrm{cm}^{-1}: 3121(\mathrm{OH}), 1621(\mathrm{C}=\mathrm{O}), 1250$ $\left(\mathrm{C}-\mathrm{OCH}_{3}\right) .{ }^{1} \mathrm{HNMR}\left(\mathrm{CDCl}_{3}\right): 3.90\left(\mathrm{~s}, 3 \mathrm{H}, \mathrm{C}-\mathrm{OCH}_{3}\right)$; $3.92\left(\mathrm{~s}, 9 \mathrm{H}, 3 \times \mathrm{OCH}_{3}\right) ; 6.96(\mathrm{~d}, 1 \mathrm{H}, \mathrm{CO}-\mathrm{CH}=\mathrm{CH})$; $7.64(\mathrm{~d}, 1 \mathrm{H}, \mathrm{CO}-\mathrm{CH}=\mathrm{CH}) ; 6.85\left(\mathrm{~s}, 2 \mathrm{H}, \mathrm{C}_{2}-\mathrm{H}_{2} \& \mathrm{C}_{6}-\right.$ H). Analysis found: $\mathrm{C}, 68.34 ; \mathrm{H}, 5.74 \%$. $; \mathrm{C}_{18} \mathrm{H}_{18} \mathrm{O}_{5}$ Required:C, 68.78; $\mathrm{H}, 5.77 \%$. 
3-(2,4-dichlorophenyl)-1-(4-hydroxyphenyl)-2propen-1-one (19)

Yield $77 \%$, m.p. $157-158^{\circ} \mathrm{C}$. IR ( $\left.\mathrm{KBr}\right)$ $\mathrm{cm}^{-1}: 3171(\mathrm{OH}), 1651(\mathrm{C}=\mathrm{O}), 710(\mathrm{C}-\mathrm{Cl})$. Analysis found : $\mathrm{C}, 61.18 ; \mathrm{H}, 3.46 ; \mathrm{Cl}, 23.64 \% ; \mathrm{C}_{15} \mathrm{H}_{10} \mathrm{Cl}_{2} \mathrm{O}_{2}$ Required:C, 61.46; H, 3.44; Cl, 24.19\%.

3-(4-bromophenyl)-1-(4-hydroxyphenyl)-2propen-1-one (20)

Yield $51 \%$, m.p. $181-182^{\circ} \mathrm{C}$ (Lit. $\left.180^{\circ} \mathrm{C}\right)^{8}$. IR (KBr) cm-1: 3147(OH), 1670(C=O), 561(C-Br). Analysis found: $\mathrm{C}, 59.53 ; \mathrm{H}, 3.53 ; \mathrm{Br}, 25.96 \% ; \mathrm{C}_{15} \mathrm{H}_{11}$ $\mathrm{Br} \mathrm{O}_{2}$ Required :C, 59.43; H, 3.66; Br, 26.36\%.

\section{3-(3,4-dimethoxyphenyl)-1-(4-hydroxyphenyl)-2-} propen-1-one (21)

Yield $67 \%$, m.p. $121-122^{\circ} \mathrm{C}$. IR $(\mathrm{KBr}) \mathrm{cm}^{-1}$ : 3100(OH), 1690(C=O), $1275\left(\mathrm{C}-\mathrm{OCH}_{3}\right) .{ }^{1} \mathrm{HNMR}$ $\left(\mathrm{CDCl}_{3}\right): 3.96\left(\mathrm{~s}, 3 \mathrm{H}, \mathrm{C}-\mathrm{OCH}_{3}\right) ; 3.98\left(\mathrm{~s}, 3 \mathrm{H}, \mathrm{C}-\mathrm{OCH}_{3}\right)$; $7.40(\mathrm{~d}, \mathrm{~J}=16.0 \mathrm{~Hz}, 1 \mathrm{H}, \mathrm{CO}-\mathrm{CH}=\mathrm{CH}) ; 7.86$ (d, $\mathrm{J}=16.0 \mathrm{~Hz}, 1 \mathrm{H}, \mathrm{CO}-\mathrm{CH}=\mathrm{CH}) ; 7.90-8.45(\mathrm{~m}, 8 \mathrm{H}, \mathrm{Ar}-$ $\mathrm{H})$. Analysis found: $\mathrm{C}, 71.96 ; \mathrm{H}, 5.64 \%$. $; \mathrm{C}_{17} \mathrm{H}_{16} \mathrm{O}_{4}$ Required :C, 71.82; $\mathrm{H}, 5.67 \%$.

3-(2,4-dimethoxyphenyl)-1-(4-hydroxyphenyl)-2propen-1-one (22)

Yield $69 \%$, m.p. $123-124^{\circ} \mathrm{C}$. IR $(\mathrm{KBr}) \mathrm{cm}$ 1: $3260(\mathrm{OH}), 1680(\mathrm{C}=\mathrm{O}), 1255,1250\left(\mathrm{C}-\mathrm{OCH}_{3}\right)$. Analysis found : $\mathrm{C}, 71.68 ; \mathrm{H}, 5.60 \% . ; \mathrm{C}_{17} \mathrm{H}_{16} \mathrm{O}_{4}$ Required: C, 71.82; $\mathrm{H}, 5.67 \%$.

\section{3-(3-bromophenyl)-1-(4-hydroxyphenyl)-2-} propen-1-one (23)

Yield $60 \%$, m.p. $164-165^{\circ} \mathrm{C}$. IR $(\mathrm{KBr}) \mathrm{cm}^{-1}$ : $3200(\mathrm{OH}), 1677(\mathrm{C}=\mathrm{O})$. Analysis found: $\mathrm{C}, 59.16 ; \mathrm{H}$, 3.45; $\mathrm{Br}, 26.29 \% ; \mathrm{C}_{15} \mathrm{H}_{11} \mathrm{BrO}_{2}$ Required : C, 59.43; $\mathrm{H}, 3.66$; $\mathrm{Br}, 26.36 \%$.

\section{3-(2-chlorophenyl)-1-(4-hydroxyphenyl)-2- propen-1-one (24)}

Yield $74 \%$, m.p. $155-156^{\circ} \mathrm{C}$. IR $(\mathrm{KBr}) \mathrm{cm}^{-1}$ : $3300(\mathrm{OH}), 1660(\mathrm{C}=\mathrm{O}), 750(\mathrm{C}-\mathrm{Cl})$ Analysis found:C, 69.69; $\mathrm{H}, 4.29 ; \mathrm{Cl}, 13.57 \% ; \mathrm{C}_{15} \mathrm{H}_{11} \mathrm{ClO}_{2}$ Required : C, 69.64; H, 4.29; Cl, 13.70\%.

\section{3-(2-methoxyphenyl)-1-(4-hydroxyphenyl)-2- propen-1-one (25)}

Yield $70 \%$, m.p. $164-166^{\circ} \mathrm{C}$. IR $(\mathrm{KBr}) \mathrm{cm}$ 1:1635(C=O), 3270(OH), $1252\left(\mathrm{C}-\mathrm{OCH}_{3}\right)$. Analysis found : $\mathrm{C}, 75.32 ; \mathrm{H}, 5.56 \%$. $\mathrm{C}_{16} \mathrm{H}_{14} \mathrm{O}_{3}$ Required:C,
75.57; H, 5.55\%.

4-[3-(4-chlorophenyl) prop-2-enoyl]phenyl phenylcarbamate (27)

A mixture of 3-(4-chlorophenyl)-1-(4hydroxyphenyl)-2-propen-1-one (14, 1gm, $4 \mathrm{mmol})$ and phenylisocyanate $(\mathbf{2 6}, 0.476 \mathrm{gm}, 4 \mathrm{mmol})$ in dry benzene $(20 \mathrm{ml})$ was refluxed for 12 hours on steam bath. Completion of the reaction was monitored by TLC. It was then concentrated under vacuum, to give solid residue which was crystallized from benzene to afford 27 . Yield $66 \%$, m.p. $191-192^{\circ} \mathrm{C}$. IR $(\mathrm{KBr}) \quad \mathrm{cm}^{-1}: 3380(\mathrm{NH}), 1690(\mathrm{NH}-\mathrm{CO}), 740(\mathrm{C}-\mathrm{Cl})$. ${ }^{1} \mathrm{HNMR}\left(\mathrm{DMSO}-\mathrm{D}_{6}\right): 7.01\left(\mathrm{~d}, \mathrm{~J}=8.0 \mathrm{~Hz}, 2 \mathrm{H}, \mathrm{C}_{3}-\mathrm{H} \&\right.$ $\left.\mathrm{C}_{5}-\mathrm{H}\right), 7.36(\mathrm{~d}, \mathrm{~J}=16.0 \mathrm{~Hz}, 1 \mathrm{H}, \mathrm{COCH}=\mathrm{CH}), 7.38$ $\left(\mathrm{d}, \mathrm{J}=8.0 \mathrm{~Hz}, 2 \mathrm{H}, \mathrm{C}_{3}-\mathrm{H} \&^{\prime} \mathrm{C}_{5}-\mathrm{H}\right), 7.52(\mathrm{~d}, \mathrm{~J}=16.0 \mathrm{~Hz}$, $1 \mathrm{H}, \mathrm{COCH}=\mathrm{CH}), 7.55\left(\mathrm{~d}, \mathrm{~J}=8.0 \mathrm{~Hz}, 2 \mathrm{H}, \mathrm{C}_{2}-\mathrm{H} \& \mathrm{C}_{6}-\mathrm{H}\right)$, $7.66\left(\mathrm{~d}, \mathrm{~J}=8.0 \mathrm{~Hz}, 2 \mathrm{H}, \mathrm{C}_{2}-\mathrm{H} \& \mathrm{C}_{6}-\mathrm{H}\right), 7.66-7.75(\mathrm{~m}$, $5 \mathrm{H}, \mathrm{Ar}-\mathrm{H}), 8.50(\mathrm{~s}, 1 \mathrm{H}, \mathrm{NH})$. Analysis found: $\mathrm{C}, 69.72$; $\mathrm{H}, 4.25$; $\mathrm{Cl}, 9.31$; N, 3.64\%.; $\mathrm{C}_{22} \mathrm{H}_{16} \mathrm{CINO}_{3}$ Required: C, 69.94; H, 4.27; Cl, 9.38; N, 3.71\%.

Other compounds 28-36 were prepared similarly from $15-23$ and 26.

\section{4-[3-(4-methylphenyl) prop-2-enoyl]phenyl phenylcarbamate (28)}

Yield $60 \%$, m.p. $192-194^{\circ} \mathrm{C}$. IR $(\mathrm{KBr}) \mathrm{cm}^{-1}$ : $3370(\mathrm{NH}), 1692(\mathrm{NH}-\mathrm{CO}), 1380\left(\mathrm{C}-\mathrm{CH}_{3}\right)$. Analysis found: C, 77.38; $\mathrm{H}, 5.33 ; \mathrm{N}, 3.89 \% ; \mathrm{C}_{23} \mathrm{H}_{19} \mathrm{NO}_{3}$ Required :C, 77.29; H, 5.36; N, 3.92\%.

\section{4-[3-(4-nitrophenyl) prop-2-enoyl]phenyl phenylcarbamate (29)}

Yield $68 \%$, m.p. $215-216^{\circ} \mathrm{C}$. IR (KBr) $\mathrm{cm}^{-1}: 3380(\mathrm{NH}), 1695$ (NH-CO), 1540, 1370 (C$\mathrm{NO}_{2}$ ). Analysis found: C, 67.95; $\mathrm{H}, 3.97 ; \mathrm{N}, 7.19 \%$ ; $\mathrm{C}_{22} \mathrm{H}_{16} \mathrm{~N}_{2} \mathrm{O}_{5}$ Required: $\mathrm{C}, 68.04 ; \mathrm{H}, 4.15 ; \mathrm{N}, 7.21 \%$.

\section{4-[3-(4-methoxyphenyl) prop-2-enoyl]phenyl phenylcarbamate (30)}

Yield $70 \%$, m.p. $147-148^{\circ} \mathrm{C}$. IR $(\mathrm{KBr})$ $\mathrm{cm}^{-1}: 3400(\mathrm{NH}), 1680(\mathrm{NH}-\mathrm{CO}), 1250\left(\mathrm{CO}-\mathrm{CH}_{3}\right)$. Analysis found: C, 73.61; H, 5.17; N, 3.29\%. ; $\mathrm{C}_{23} \mathrm{H}_{19} \mathrm{NO}_{4}$ Required: C, 73.98; $\mathrm{H}, 5.13 ; \mathrm{N}, 3.75 \%$.

\section{4-[ 3-(3,4,5-trimethoxyphenyl)prop-2-enoyl] phenyl phenylcarbamate (31)}

Yield $66 \%$, m.p. $149-150^{\circ} \mathrm{C}$. IR $(\mathrm{KBr})$ $\mathrm{cm}^{-1}$ : $3365(\mathrm{NH}), 1690(\mathrm{NH}-\mathrm{CO}), 1200\left(\mathrm{C}-\mathrm{OCH}_{3}\right)$. 
${ }^{1} \mathrm{HNMR}\left(\mathrm{DMSO}-\mathrm{D}_{6}\right.$ ): $3.86\left(\mathrm{~s}, 9 \mathrm{H}, 3 \mathrm{xOCH}_{3}\right) ; 6.97$ $(\mathrm{d}, \mathrm{J}=16.0 \mathrm{~Hz}, 1 \mathrm{H}, \mathrm{COCH}=\mathrm{CH}), 7.64(\mathrm{~d}, \mathrm{~J}=16.0 \mathrm{~Hz}, 1 \mathrm{H}$, $\mathrm{COCH}=\mathrm{CH}), 7.78-8.30(\mathrm{~m}, 9 \mathrm{H}, \mathrm{Ar}-\mathrm{H}), 8.50(\mathrm{~s}, 1 \mathrm{H}$, $\mathrm{NH}$ ). Analysis found: C, 69.69; H, 5.37; N, 3.17\%. ; ${ }_{25} \mathrm{H}_{23} \mathrm{NO}_{6}$ Required: $\mathrm{C}, 69.27 ; \mathrm{H}, 5.35 ; \mathrm{N}, 3.23 \%$.

\section{4-[3-(2,4-dichlorophenyl)prop-2-enoyl]phenyl phenylcarbamate (32)}

Yield $72 \%$, m.p. $225-226^{\circ} \mathrm{C}$. IR $(\mathrm{KBr}) \mathrm{cm}^{-}$ 1: $3380(\mathrm{NH}), 1680$ (NH-CO), 760 (C-Cl). Analysis found: C, 63.86; H, 3.64; Cl, 17.22; N, 3.29\%. $\mathrm{C}_{22} \mathrm{H}_{15} \mathrm{Cl}_{2} \mathrm{NO}_{3}$ Required: $\mathrm{C}, 64.09 ; \mathrm{H}, 3.67 ; \mathrm{Cl}, 17.20$; $\mathrm{N}, 3.40 \%$.

\section{4-[3-(4-bromophenyl) prop-2-enoyl]phenyl} phenylcarbamate (33)

Yield $58 \%$, m.p. $196-198^{\circ} \mathrm{C}$. IR(KBr) $\mathrm{cm}^{-1}: 3390(\mathrm{NH}), 1675$ (NH-CO), 540,520 (C-Br). Analysis found: $\mathrm{C}, 62.76 ; \mathrm{H}, 3.84 ; \mathrm{Br}, 18.73 ; \mathrm{N}, 3.19 \%$. $; \mathrm{C}_{22} \mathrm{H}_{16} \mathrm{BrNO}_{3}$ Required:C, 62.57; $\mathrm{H}, 3.82 ; \mathrm{Br}, 18.92$; $\mathrm{N}, 3.32 \%$.

\section{4-[3-(3,4-dimethoxyphenyl) prop-2-enoyl]phenyl} phenylcarbamate (34)

Yield $65 \%$, m.p. $198-199^{\circ} \mathrm{C}$. IR $(\mathrm{KBr})$

$\mathrm{cm}^{-1}: 3400(\mathrm{NH}), 1695(\mathrm{NH}-\mathrm{CO}), 1255\left(\mathrm{C}-\mathrm{OCH}_{3}\right)$. ${ }^{1} \mathrm{HNMR}\left(\mathrm{DMSO}-\mathrm{D}_{6}\right): 3.76$ (s, $\left.3 \mathrm{H}, \mathrm{OCH}_{3}\right) ; 3.80$ (s, $3 \mathrm{H}$, $\left.\mathrm{OCH}_{3}\right) ; 6.95(\mathrm{~d}, \mathrm{~J}=16.0 \mathrm{~Hz}, 1 \mathrm{H}, \mathrm{COCH}=\mathrm{CH}), 7.62(\mathrm{~d}$, $\mathrm{J}=16.0 \mathrm{~Hz}, 1 \mathrm{H}, \mathrm{COCH}=\mathrm{CH}), 7.79-8.30(\mathrm{~m}, 1 \mathrm{H}, \mathrm{Ar}-$ $\mathrm{H}), 8.45(\mathrm{~s}, 1 \mathrm{H}, \mathrm{NH})$. Analysis found : $\mathrm{C}, 71.23 ; \mathrm{H}$, 5.24; N, 3.26\%. ; $\mathrm{C}_{24} \mathrm{H}_{21} \mathrm{NO}_{5}$ Required: $\mathrm{C}, 71.45 ; \mathrm{H}$, $5.25 ; \mathrm{N}, 3.47 \%$.

\section{4-[3-(2,4-dimethoxyphenyl) prop-2-enoyl]phenyl phenylcarbamate (35)}

Yield $62 \%$, m.p. $220-221^{\circ} \mathrm{C}$. IR $(\mathrm{KBr}) \mathrm{cm}^{-1}$ : $3380(\mathrm{NH}), 1690(\mathrm{NH}-\mathrm{CO}), 1250\left(\mathrm{C}-\mathrm{OCH}_{3}\right)$. Analysis found: $\mathrm{C}, 71.39 ; \mathrm{H}, 5.22 ; \mathrm{N}, 3.21 \% ; \mathrm{C}_{24} \mathrm{H}_{21} \mathrm{NO}_{5}$ Required : C, $71.45 ; \mathrm{H}, 5.25 ; \mathrm{N}, 3.47 \%$.

\section{4-[3-(3-bromophenyl) prop-2-enoyl]phenyl phenylcarbamate (36)}

Yield $56 \%$, m.p. $223-225^{\circ} \mathrm{C}$. IR $(\mathrm{KBr})$ $\mathrm{cm}^{-1}: 3388(\mathrm{NH}), 1670(\mathrm{NH}-\mathrm{CO}), 540(\mathrm{C}-\mathrm{Br})$. Analysis found: $\mathrm{C}, 62.76 ; \mathrm{H}, 3.84 ; \mathrm{Br}, 18.73 ; \mathrm{N}$, 3.19\%.; $\mathrm{C}_{22} \mathrm{H}_{16} \mathrm{BrNO}_{3}$. Required: $\mathrm{C}, 62.57 ; \mathrm{H}, 3.82 ; \mathrm{Br}$, $18.92 ; \mathrm{N}, 3.32 \%$.

\section{Nematicidal Bioevaluation}

The plant parasitic nematode Meloidogyne javanica (Treub) Chitwood was used as test organism. Stock solutions of $2000 \mathrm{mg} \mathrm{L}^{-1}$ of all the compounds were prepared by dispersing these in acetone. Nematicidal activity was evaluated against second stage juveniles $\left(\mathrm{J}_{2}\right)$ of $M$. javanica. A suspension of juveniles $(1 \mathrm{ml})$ was poured into $5 \mathrm{~cm}$ Petri-dishes. Measured quantities of stock solution were added to these Petri-dishes to make final concentrations of $1000,500,250$ and $125 \mathrm{ppm}$. Acetone with water was used as control. No nematode mortality was recorded in these controls, and is therefore not included in the table. Each treatment was replicated three times. These Petri-dishes were kept in a BOD incubator at $28 \pm 1^{\circ} \mathrm{C}$. Observations were recorded after $24 \mathrm{~h}$ and $48 \mathrm{~h}$ by counting live (active) and dead (inactive) $\mathrm{J}_{2} \mathrm{~s}$ under a stereoscopic binocular microscope and the per cent mortality was counted ${ }^{10}$. The revival of immobilized nematodes was examined by randomly transferring ten $\mathrm{J}_{2} \mathrm{~s}$ to water for $24 \mathrm{~h}$. None of those immobilized $\mathrm{J}_{2} \mathrm{~s}$ revived. The experimental data was statistically analyzed using two factorial completely randomized design; the compounds and the concentrations constituting the two factors.

\section{RESULTS AND DISCUSSION}

The condensation of 4-chloro/methyl/nitro/ methoxy/3,4,5-trimethoxy/2,4-dochloro/4-bromo/3,4dimethoxy/2,4-dimethoxy/3-bromo/2-chloro/2methoxy benzaldehyde (1-12) with 4-hydroxy acetophenone (13) in using $20 \%$ ethanolic sodium hydroxide after stirring gave the corresponding 3-(4-chloro/ methyl/ nitro/ methoxy/ 3,4,5-trimethoxy/ 2,4-dichloro/ 4-bromo/3,4-dimethoxy/2,4dimethoxy/3-bromo phenyl)-1-(4-hydroxy phenyl)2-prop-1-one (14-25). Thus, a total of twelve different chalcones were synthesized. The different benzaldehyde derivatives used in this reaction are listed in Scheme (Scheme-I).

The ${ }^{1}$ HNMR spectra of $14-25$ were in accordance with the proposed structures. The substituted-2-propen-1-ones were found to possess E-configuration, which is confirmed on the basis of their ${ }^{1} \mathrm{H}$ NMR spectra. The diagnostic olefinic protons (- $\mathrm{CO}-\mathrm{CH}=\mathrm{CH}-$ ) attached to carbonyl functionality appeared as clean doublets at around 7.42 and $7.67 \delta$ (each with $\mathrm{J}=16.0 \mathrm{~Hz}$ ), situated at $\alpha$ and $\beta$ respectively and this confirms the condensation of the reactants. Methyl, methoxy and trimethoxy 
<smiles>[R]c1cc(C=O)c([R])c([R])c1[R]</smiles>

$1-12$<smiles>CC(=O)c1ccc(O)cc1</smiles>

13<smiles>[Z20]c1cc(/C=C/C(=O)c2ccc(O)cc2)c([Z2])c([R4])c1[Z2]</smiles>

$14-25$

Reagents and reaction conditions $\mathrm{NaOH}(20 \%)$, Ethanol, Stirring

\begin{tabular}{lllll}
\hline Compd. & $\mathbf{R}^{1}$ & $\mathbf{R}^{2}$ & $\mathbf{R}^{3}$ & $\mathbf{R}^{4}$ \\
\hline$\underline{1}, \underline{14}$ & $\mathrm{H}$ & $\mathrm{H}$ & $\mathrm{Cl}$ & $\mathrm{H}$ \\
$\underline{2}, \underline{15}$ & $\mathrm{H}$ & $\mathrm{H}$ & $\mathrm{CH}_{3}$ & $\mathrm{H}$ \\
$\underline{3}, \underline{16}$ & $\mathrm{H}$ & $\mathrm{H}$ & $\mathrm{NO}_{2}$ & $\mathrm{H}$ \\
$\underline{4}, \underline{17}$ & $\mathrm{H}$ & $\mathrm{H}$ & $\mathrm{OCH}_{3}$ & $\mathrm{H}$ \\
$\underline{5}, \underline{18}$ & $\mathrm{H}$ & $\mathrm{OCH}_{3}$ & $\mathrm{OCH}_{3}$ & $\mathrm{OCH}_{3}$ \\
$\underline{6}, \underline{19}$ & $\mathrm{Cl}$ & $\mathrm{H}$ & $\mathrm{Cl}$ & $\mathrm{H}$ \\
$\underline{7}, \underline{20}$ & $\mathrm{H}$ & $\mathrm{H}$ & $\mathrm{Br}$ & $\mathrm{H}$ \\
$\underline{8}, \underline{21}$ & $\mathrm{H}$ & $\mathrm{OCH}_{3}$ & $\mathrm{OCH}_{3}$ & $\mathrm{H}$ \\
$\underline{9}, \underline{22}$ & $\mathrm{OCH}_{3}$ & $\mathrm{H}$ & $\mathrm{OCH}_{3}$ & $\mathrm{H}$ \\
$\underline{10}, \underline{23}$ & $\mathrm{H}$ & $\mathrm{Br}$ & $\mathrm{H}$ & $\mathrm{H}$ \\
$\underline{11}, \underline{24}$ & $\mathrm{H}$ & $\mathrm{Cl}$ & $\mathrm{H}$ & $\mathrm{H}$ \\
$\underline{12}, \underline{25}$ & $\mathrm{H}$ & $\mathrm{OCH}_{3}$ & $\mathrm{H}$ & $\mathrm{H}$
\end{tabular}

Scheme 1: Synthesis of 3-(substitutedphenyl)-1-(4-hydroxyphenyl)-2-propen-1-ones

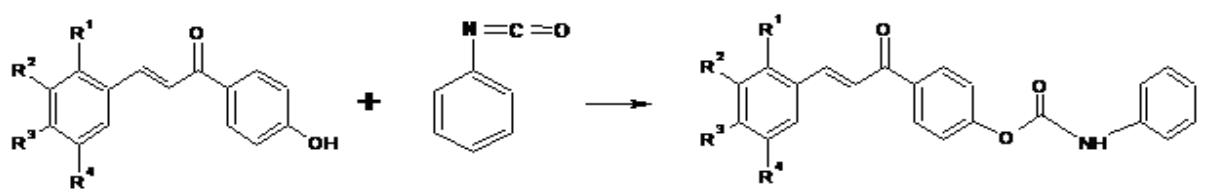

$14-23$

26

Reagents and reaction conditions:

Benzene, $\Delta$

\begin{tabular}{lllll}
\hline Compd. & $\mathbf{R}^{1}$ & $\mathbf{R}^{2}$ & $\mathbf{R}^{3}$ & $\mathbf{R}^{4}$ \\
\hline 27 & $\mathrm{H}$ & $\mathrm{H}$ & $\mathrm{Cl}$ & $\mathrm{H}$ \\
28 & $\mathrm{H}$ & $\mathrm{H}$ & $\mathrm{CH}_{3}$ & $\mathrm{H}$ \\
29 & $\mathrm{H}$ & $\mathrm{H}$ & $\mathrm{NO}_{2}$ & $\mathrm{H}$ \\
30 & $\mathrm{H}$ & $\mathrm{H}$ & $\mathrm{OCH}_{3}$ & $\mathrm{H}$ \\
31 & $\mathrm{H}$ & $\mathrm{OCH}_{3}$ & $\mathrm{OCH}_{3}$ & $\mathrm{OCH}_{3}$ \\
32 & $\mathrm{Cl}$ & $\mathrm{H}$ & $\mathrm{Cl}$ & $\mathrm{H}$ \\
33 & $\mathrm{H}$ & $\mathrm{H}$ & $\mathrm{Br}^{2}$ & $\mathrm{H}$ \\
34 & $\mathrm{H}$ & $\mathrm{OCH}_{3}$ & $\mathrm{OCH}_{3}$ & $\mathrm{H}$ \\
35 & $\mathrm{OCH}_{3}$ & $\mathrm{H}$ & $\mathrm{OCH}_{3}$ & $\mathrm{H}$ \\
36 & $\mathrm{H}$ & $\mathrm{Br}$ & $\mathrm{H}$ & $\mathrm{H}$ \\
\hline
\end{tabular}

Scheme 2: Synthesis of 4-[3-(substitutedphenyl)prop-2-enoyl]phenyl phenyl carbamates 


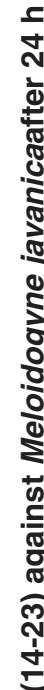
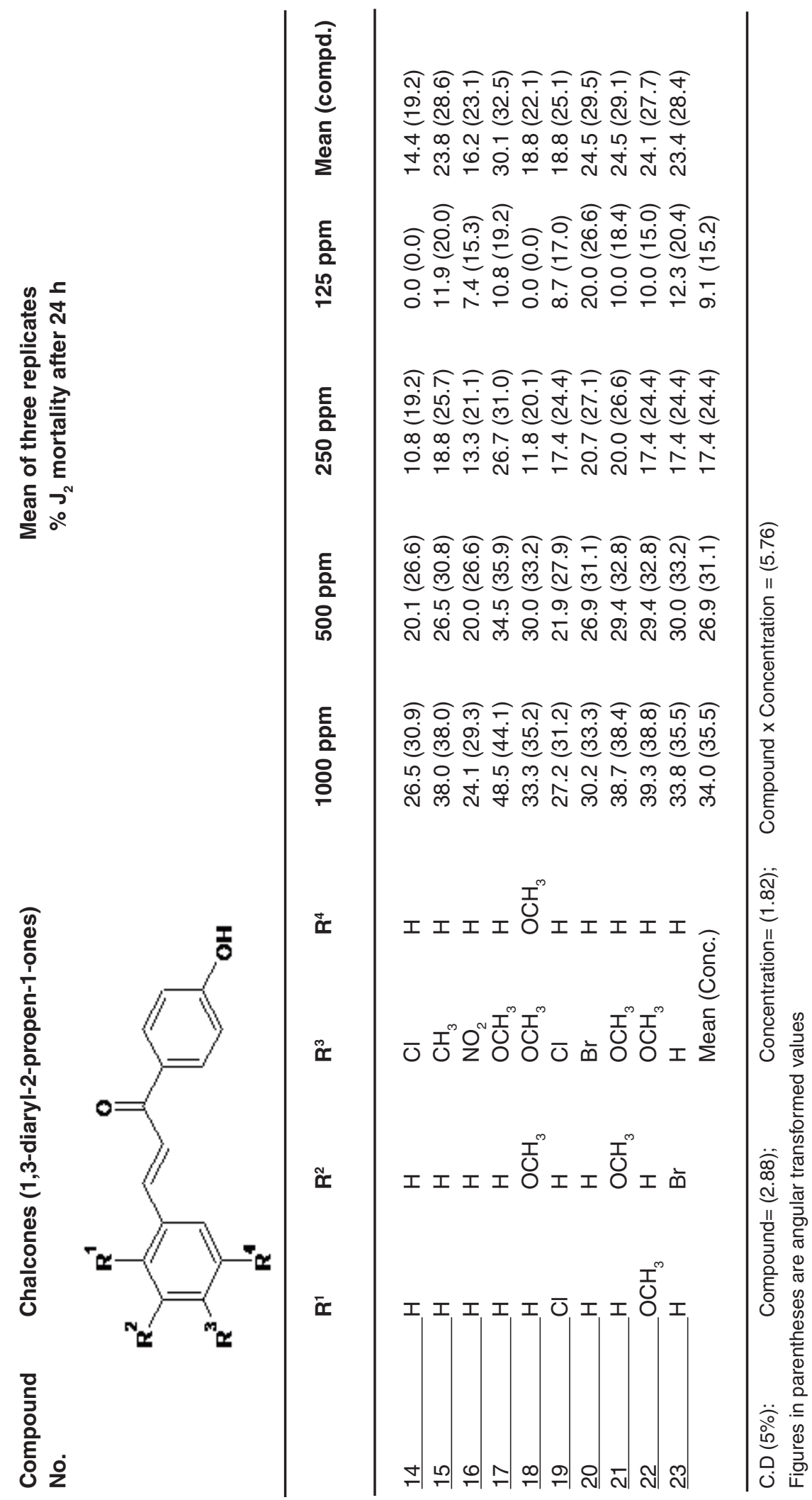


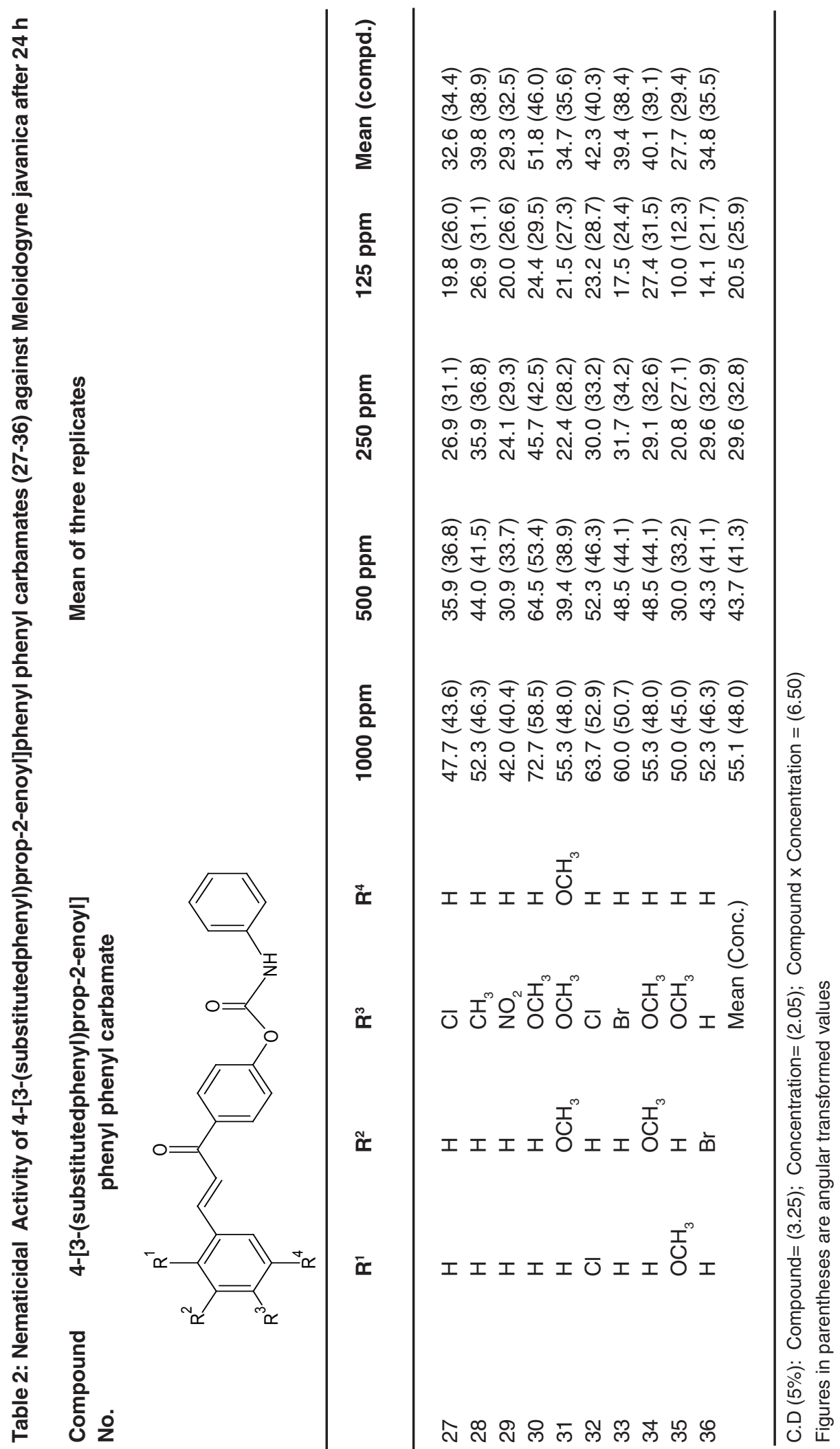


moieties could be picked up at around $2.38 \delta, 3.83$ $\delta$ and $3.91 \delta$ as singlets, each integrating three and nine protons. The $A_{2} B_{2}$ pattern of aromatic protons in the NMR spectra of compounds possessing para methyl phenyl moiety, could be picked up as ortho coupled doublets at about $7.01 \delta$ (ortho to methyl) and $7.50 \delta$ (ortho to $-\mathrm{CH}=\mathrm{CH}$ - group), each integrating for two protons with $\mathrm{J}=8.0 \mathrm{~Hz}$. Similar pattern was also observed in para chloro or bromo moieties, $7.70 \delta$ due deshielding effect of carbonyl functionalities.

In the ${ }^{1}$ HNMR spectrum of 3-(4-chlorophenyl)-1-(4-hydroxyphenyl)-2-propen1-one (14), a doublet at 7.19 ' integrating for two protons $(\mathrm{J}=8.0 \mathrm{~Hz})$ was observed, which confirm the two aromatic protons of $\mathrm{C}_{3}$ and $\mathrm{C}_{5}$ positions (ortho to chloro) respectively. The two diagnostic doublets $(\mathrm{J}=16.0 \mathrm{~Hz})$ could be assigned at $7.42^{\prime}$ and 7.74 ' respectively for $-\mathrm{CO}-\mathrm{CH}=\mathrm{CH}$ - protons ( \pm and 2 to keto) was indication of two olefinic protons. A doublet integrating for two protons with $\mathrm{J}=8.0 \mathrm{~Hz}$ was observed at $7.67^{\prime}$ for $C_{3}$ ' \& $C_{5}$ ' respectively while $C_{2}$ and $\mathrm{C}_{6}$ protons were picked up as doublet at $7.69 \delta$ $(\mathrm{J}=8.0 \mathrm{~Hz})$. A methoxy group was observed as singlet at $3.83 \delta$ and all other protons at their usual positions in 3-(4-methoxyphenyl)-1-(4-hydroxyphenyl)-2propen-1-one (17) supported the assigned structure. Appearance of peaks near $1680 \mathrm{~cm}^{-1}$ and 3300 $\mathrm{cm}^{-1}$ for $\mathrm{C}=\mathrm{O}$ stretching and $\mathrm{OH}$ functionality, in the IR spectra of the above compounds further corroborated their structures.

In the next step of synthesis, condensation of compounds 3-(4-chloro/ methyl/ nitro/ methoxy/ 3,4,5-trimethoxy/ 2,4-dichloro/ 4-bromo/3,4dimethoxy/2,4-dimethoxy/3-bromo phenyl)-1(4-hydroxy phenyl)-2-prop-1-one (14-25) with phenylisocyanate (26) in equimolar ratio in dry benzene by refluxing on steam bath for $12 \mathrm{~h}$, afforded their corresponding 4[3-(4-chloro/methyl/nitro/ methoxy/3,4,5-trimethoxy/2,4-dichloro/4-bromo/3,4dimethoxy/2,4-dimethoxy/3-bromo phenyl) prop2-enoyl] phenyl phenylcarbamate (27-36) in good yields.(Scheme-II)

Thus, a total of ten different 4-[3-(substitutedphenyl)prop-2-enoyl]phenyl phenyl carbamates were synthesized. The different 3-(substitutedphenyl)-1-(4-hydroxyphenyl)-2propen-1-ones used in this reaction are listed in
scheme-II.

In the ${ }^{1}$ HNMR spectrum of 4-[3-(4-chlorophenyl) prop-2-enoyl] phenyl phenyl carbamate (27), two aromatic protons at positions 3 and 5 appeared at $7.01 \delta(\mathrm{J}=8.0 \mathrm{~Hz})$ as doublet. Another two doublets at 7.38 ' and $7.55^{\prime}$ integrating for two protons each were assigned to protons at positions $\mathrm{C}_{3}$ ' and $\mathrm{C}_{5}$ ' or $\mathrm{C}_{2}$ and $\mathrm{C}_{6}$ respectively. The downfield shift of $\mathrm{C}_{2}$ ' and $\mathrm{C}_{6}$ ' protons ortho to carbonyl functionalities appeared at $7.66 \delta$. The remaining aromatic protons appeared as multiplet at $7.66 \delta$ to $7.55 \delta$. The broad singlet at $8.50 \delta$ was assigned to $\mathrm{NH}$ proton of carbamoyloxy moiety. The formation of carbamates of the above compounds follows from the mode of synthesis and were supported by the appearance of a band around $3340 \mathrm{~cm}^{-1}$ for $\mathrm{NH}$ functionality and around 1670 and $1690 \mathrm{~cm}^{-1}$ for carbonyl and carbamoyloxy $(\mathrm{C}=\mathrm{O})$ functionalities respectively. Thus, structures of all these compounds were fully supported by their NMR and IR spectra.

\section{Nematicidal Bioevaluation}

The mortality of $M$. javanica $\mathrm{J}_{2} \mathrm{~s}$ after $24 \mathrm{~h}$ by various 3-(substitutedphenyl)-1-(4-hydroxyphenyl)2-propen-1-one has been given in table 1. Overall moderate activity was shown by 3-(4-chloro/ methyl/ nitro/methoxy/3, 4, 5-trimethoxy/2, 4-dichloro/4bromo/3, 4-dimethoxy/2, 4-dimethoxy/3-bromo phenyl)-1-(4-hydroxyphenyl)-2-propen-1-one(14-23) compounds. The perusal of the activity data revealed that interaction of compounds and concentrations was statistically significant. Irrespective of the concentration, compound 3-(4-methoxyphenyl)-1(4-hydroxyphenyl)-2-propen-1-one (17) have shown maximum nematode mortality i.e. $30 \%$ and it is highest activity of this series. This was followed by compounds 15, 20, 21, 22 and 23, which resulted in the range of $29.5-27.7 \%$ and were found statistically at par. Compounds 16, 18 and 19 were relatively less active. The compound 3-(4-chlorophenyl)-1-(4hydroxyphenyl)-2-propen-1-one (14) was found least active.

In general, nematoxicity increased with time. Among these compounds no significant difference was recorded after 48h. Compound 17, 22 and 21 exhibited highest toxicity at 1000ppm and compound 14, 18, 16 and 19 showed least 
toxicity at $125 \mathrm{ppm}$ and were statistically at par. Replacement of chloro with bromine, methyl and methoxy at position-3 increased the nematicidal activity. Presence of dimethoxy groups also showed appreciable activity. Unexpectedly, nematicidal activity decreased by increasing number of methoxy groups as in case of compound 3-(3,4,5-trimethoxy phenyl)-1-(4-hydroxyphenyl)-2-propen-1-one (18). Irrespective of compounds, 1000ppm concentration proved to be most toxic to nematodes and $125 \mathrm{ppm}$ concentration was found least effective. However, all the concentrations were significantly different from one another.

In this series, compounds 4-[3-(substitutedphenyl)prop-2-enoyl]phenyl phenyl carbamates (27-36) were synthesized from 3-(substitutedphenyl)-1-(4-hydroxyphenyl)-2propen-1-ones (14-23) and selected for nematicidal activity in vitro. There was an overall increase in the nematicidal activity as compared to starting material 3-(substitutedphenyl)-1-(4-hydroxyphenyl)2-propen-1-ones (14-23). Due to incorporation of carbamoyloxy moiety in 2-propen-1-ones enhanced the activity but not up to the desired value. Table-2, shows the data on the mortality of $M$. javanica $\mathrm{J}_{2} \mathrm{~s}$ after $24 \mathrm{~h}$ by various 4-[3-(substitutedphenyl)-prop2-enoyl]phenyl phenyl carbamate (27-36). The perusal of the activity data revealed that interaction of compounds and concentrations was statistically significant. Irrespective of the concentration, in this series compound 4-[3-(4-methoxyphenyl) prop-2enoyl] phenyl phenyl carbamate (30) have shown maximum nematode mortality i.e. $51.8 \%$ and it was comparatively highest. This was followed by compounds 28, 32, 33, and 34, which resulted in a range of $42.2-39.1 \%$ and were statistically at par. Compounds 27, 29, 31 and $\mathbf{3 6}$ were relatively less active. The compound 4-[3-(2,4-dimethoxyphenyl) prop-2-enoyl]phenyl phenylcarbamate (35) was least active and significantly, low toxicity of $35 \%$ was shown by this compound.

Among these compounds nematicidal activity increased after $48 \mathrm{~h}$. Compound 4-[3-(4-methoxyphenyl) prop-2-enoyl]phenyl phenylcarbamate (30) and 4-[3-(2,4-dichlorophenyl) prop-2-enoyl]phenyl phenylcarbamate (32) showed highest toxicity $72 \%$ at $1000 \mathrm{ppm}$ and were at par with 500 ppm concentration, followed by compounds 28, 29, 31, 33, 34, 35 and 36 which showed moderate toxicity and were statistically at par. Further introduction of carbamoyloxy moiety in these compounds resulted in higher nematicidal activity. Replacement of chloro with bromine, methyl and methoxy at position-3 increased the nematicidal activity. Compound containing chloro moiety at paraposition was found least effective while dichloro moiety significantly increased the nematicidal activity. Irrespective of compounds, concentration 1000 ppm proved to be most toxic to nematode. However, all the concentrations were significantly different from one another.

Observations on nematode mortality were also recorded after $48 \mathrm{~h}$; however since there was no significant increase in mortality, the data is not reproduced in table.

\section{ACKNOWLEDGEMENTS}

The authors are thankful to the Professor and Head, Department of Chemistry \& Physics and Department of Nematology, CCS Haryana Agricultural University, Hisar for providing laboratory facilities. Thanks also to SAIF, Panjab University, Chandigarh, for providing the spectral data. The financial help in the form of scholarship (POSE), received from DST, Panchkula, is thankfully acknowledged.

\section{REFERENCES}

1. Hague, N.G.M. and Gowen, S.R. Chemical Control of Nematodes. In principles and practice of nematode control in crops. Academic Press, 1987.

2. Prasad, Y.R.; Rao A.L. and Rambabu, R.
Synthesis and Antimicrobial Activity of some Chalcone Derivatives. E Journal of Chemistry,2008, 5(3), 461466.

3. Baron, R.L. Handbook of Pesticide Toxicology, Academic Press, New York, 1991, 1125-1189. 
4. Roy, K.K.; Dixit, A. and Saxena, A.K. J. Mol. Graph. Model., 2008, 27, 197-208.

5. Chopra, I.; Walia, R.K. and Singh, R. Pestic. Res. J., 2006, 18(2), 124-128.

6. Singh, R., Abrol, V.; Gupta, B.B. and Malik, O.P. Pestic. Sci., 1988, 20, 125-130.

7. Kumari, S.; Singh, R.; Kumar, A and Walia, R.K. Asian J. Chem., 2014, 26(11), 31393143.

8. Nikhil, Synthesis of potential agrochemicals and their evaluation against American bollworm and root-knot nematode. PhD Thesis, CCS Harayana Agricultural University, Hisar, 2006.

9. Syam, S.; Abdelwahab, S.I.; Al-Mamary, M.A. and Mohan, S. Molecules, 2012, 17, 61796195.

10. Gill,K.; Mehta,S.K.; Malik, M.S.; Malik, O.P. and Walia, R.K. Nematol. medit., 2001, 29, 219-222. 